www.jmscr.igmpublication.org Impact Factor (SJIF): 6.379

Index Copernicus Value: 71.58

ISSN (e)-2347-176x ISSN (p) 2455-0450

crossref DOI: _https://dx.doi.org/10.18535/jmscr/v6i5.47

Journal Of Medical Science And Clinical Research

IGM Publication

An official Publication of IGM Publication

\title{
Assessment of Neurophysiological variables in terms of Event Related Potentials for Intention to Masquerade Truth
}

\author{
Authors \\ Umesh Gupta $^{1}$, Abhishek Saini ${ }^{*}$, Amitabh Dube ${ }^{3}$ \\ ${ }^{1} 3^{\text {rd }}$ Year Resident, ${ }^{2}$ Assistant Professor, ${ }^{3}$ Senior Professor \\ Department of Physiology, SMS Medical College, Jaipur, India \\ *Corresponding Author
}

Abhishek Saini

Assistant Professor, Department of Physiology, S.M.S. Medical College, Jaipur, India

Phone: +91-9468651414, Email: drabhi16@gmail.com

\begin{abstract}
Background: The aim of the present study was to investigate whether Event Related Potentials (ERPs) can reveal a veiled and covert psychological process and /or inscription, an observation that underscores masquerading of an identified fact.

Objectives: To assess and compare P300 amplitude among two groups namely, deception (wherein the subjects were concealing the information) and control group (wherein the subjects were not concealing), in response towards performance of a tailor-made task that required selection of a playing card among five other cards.

Material and Methods: A total of 30 subjects in the age group of $18-25$ years participated in the present study, taking part in the formation of both groups which differed only with respect to the task requirements. The candidates were asked to select one playing card (probe) among five playing cards, which were then presented to the subject one by one in a pseudo-randomised sequence on a computer monitor, in a dimly lit, sound attenuated room. While performing under the deception group, the participants were asked to conceal the selected card from the knowledge of the examiner and to respond equivocally/uniformly towards the selected card (probe) as well towards the non-selected cards. A playing card with an image of joker served as target which required a specific response in form of pressing YES on a keyboard in order to ascertain attention. Electroencephalogram (EEG) was recorded simultaneously along with the performance of these tasks using 10-20 international system of electrode placement. The data so obtained was selectively averaged for Pz electrode site and P300 ERP component was evaluated in terms of amplitude in $\mu V$. The obtained parameters were compared between both groups and a p-value of $<0.05$ was accepted as a marker of significance.
\end{abstract}

Result: The results of the present study indicated that the amplitude of P300 component of ERP came out to be higher under the circumstances wherein the participants were performing the task under Deception group as compared to their performance as controls.

Conclusion: The P300 component of the Event Related Potentials (ERPs) can serve as a proxy marker to reveal a hidden psychological process like concealment.

Keywords: P300, Event related potentials (ERP), Electroencephalography (EEG), Deception and Concealment. 


\section{Introduction}

Deception has been considered as a neuropsychophysiological process wherein deliberate attempts are made by an individual to create an aura of belief in space time with the intension to mislead and towards which, the communicator himself or herself considers themselves as not been true (Vrij, 2000).

\section{Electroencephalography and P300}

In 1924 Hans Berger, a German psychiatrist, observed synchronised neural activity changes in reference to the functional status of the human brain (Berger, 1929). In the prototypical ERP trace, the most prominent and most explored is the P300 (P3). P300 appears when a subject detects an informative task-related stimulus. P300 reflects memory updating (Donchin and Coles, 1988). P300 amplitude is related to stimulus probability, stimulus significance, task difficulty, motivation, and vigilance. $\mathrm{P} 3$ wave is evoked by a task known as the odd-ball paradigm (visual or auditory) (Johnson, 1988)

\section{Material and Methods}

The present study was conducted in Department of Physiology, S.M.S Medical College, Jaipur, after receiving the desired clearance from the Institutional Ethical committee, on 30 willing and cooperative healthy subjects between the age group of 18-25 years, taken from various Medical and Para - Medical courses students with normal or corrected eye sight. The study design was Institution based case control type of Observational study. Those subjects suffering from any acute or chronic illness or alcoholic and/or smokers or who did not give their consent were excluded from the study.

After explaining the test procedure, the participants were asked to steal one playing card among the five playing cards as except the joker. Hereafter, the stolen card was considered as probe/relevant card and the rest of the not-stolen cards were considered as irrelevant cards. Joker served as target. For the performance of the task as under the deception group, the subjects were asked to hide the probe/relevant card from the knowledge of the examiner and also they were told beforehand, that the relevant card would elicit a larger brain electrical response as compared to that for the irrelevant cards and that the experimenter will attempt to detect the stolen card according to this information. So in this way, under the Deception task, the subjects were provided with an intention to conceal the information and thus required an active attempt to deceive the examiner.

For the performance of the task as under control group, the participants were asked to reveal the relevant card to the examiner beforehand and hence had no intention to masquerade the relevant card from the knowledge of the examiner. Raw EEG was recorded using Brain Electro Scan System (BESS) version 4.0 (Axxonet Systems Technologies Ltd., India).

The data so collected was subjected to statistical analysis through SPSS, version 21 for Windows Statistical Software Package (SPSS inc., Chicago, IL, USA) and the quantitative data was presented as mean and standard deviation and were compared by student t-test.

\section{Observation and Results}

\section{Table 1}

\begin{tabular}{|l|c|c|c|c|c|c|c|}
\hline \multirow{2}{*}{ Electrodes } & \multirow{2}{*}{ Group } & \multicolumn{2}{|c|}{ Target } & \multicolumn{2}{c|}{ Non Selected } & \multicolumn{2}{c|}{ Selected } \\
\cline { 3 - 8 } & & Mean & SD & Mean & SD & Mean & SD \\
\hline \multirow{2}{*}{ Pz } & Deception & 9.34 & 3.60 & 9.06 & 2.48 & 12.21 & 2.12 \\
\cline { 2 - 8 } & Control & 10.32 & 3.88 & 9.93 & 3.27 & 10.45 & 3.33 \\
\hline & p-value & \multicolumn{2}{|c|}{0.312} & \multicolumn{2}{|c|}{0.253} & \multicolumn{2}{c|}{0.018} \\
\hline
\end{tabular}

Table No. 1: Mean \pm SD values of Amplitude of P300 Wave Form (in Microvolts, $\mu \mathrm{V}$ ) at Pz EEG Selected Cards in Deception Group and Control Group with respective 'p' values.

Electrode Sites for Target, Non - Selected and 
Figure 1

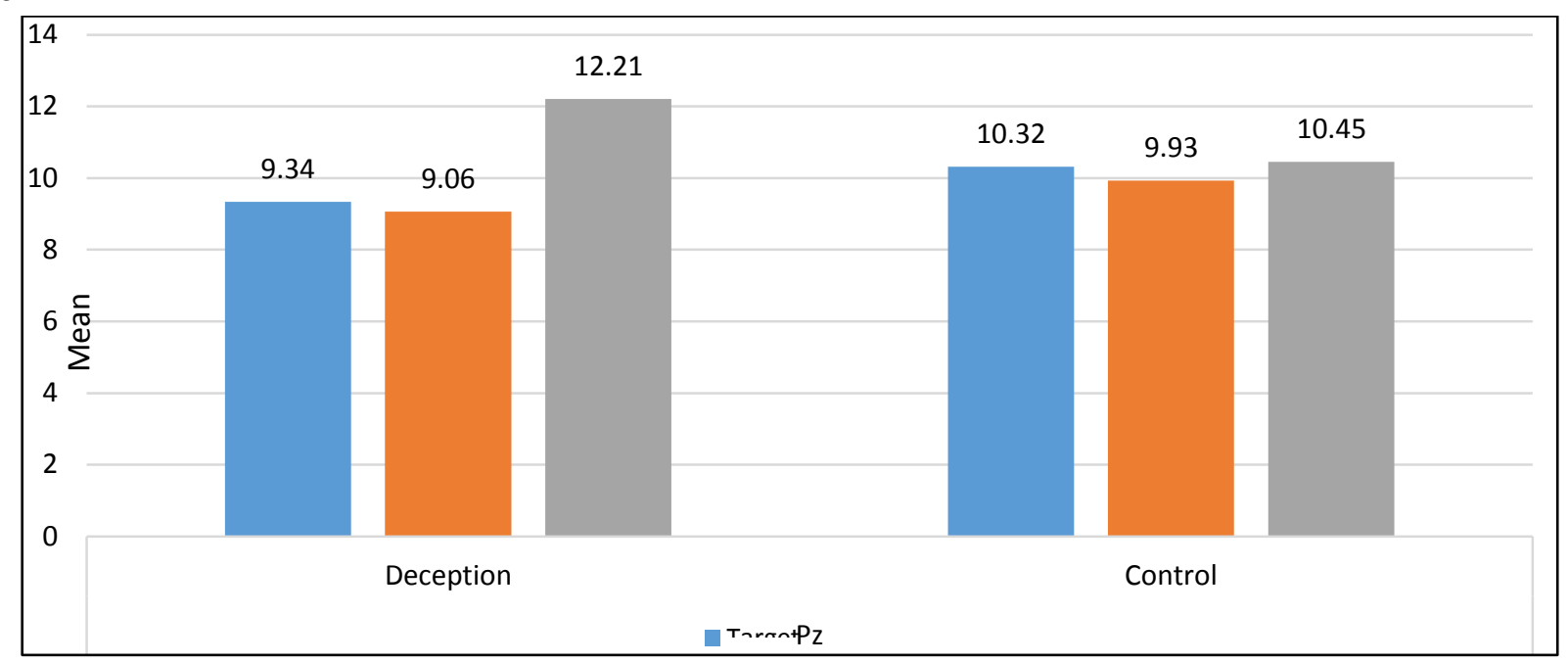

Figure: 1 exhibits graphical representation of Mean $\pm(\mathrm{SD})$ values of Amplitude of P300 Wave (in $\mu \mathrm{V}$ ) at Pz Electrodes for Target, Non - Selected and Selected Cards in Deception Group and Control Group.

\section{Results}

Table No.1 represents Mean \pm SD Values of Amplitude of $\mathrm{P} 300$ Wave Form (in $\mu \mathrm{V}$ ) at $\mathrm{Pz}$ EEG Electrode Sites for Target, Non - Selected and Selected Cards in Deception Group and Control Group. It could be observed that the values of amplitude of P300 wave form were high in the selected card manoeuvre as compared to that in other tasks of Target and Non - Selected cards in Pz EEG electrode sites and the difference in values of amplitude of the P300 at Pz differed significantly with values of $12.21 \pm 2.12 \mu \mathrm{V}$ (deception group) and $10.45 \pm 3.33 \mu \mathrm{V}$ (control group), respectively ( $\mathrm{p}$ value $=0.018$ ).

\section{Discussion}

P300 amplitude gives information on amount of attention resources allocated to stimulus, working memory and task's complexity and is related to decision making and memory processing (Hillyard, Hink, Schwent \& Picton, 1973). The values of the amplitude of P300 wave form increased significantly $(\mathrm{p}<0.05$, with $\mathrm{p}=0.018$ ) in the deception group in the $\mathrm{Pz}$ electrode site as compared to that observed in the control group. The intention to conceal the selected card paradoxically made the card more significant, and this process was reflected in a larger amplitude value of $\mathrm{P} 300$. The observations suggest and extrapolate that the endogenous ERP component in the form of P300 wave could reveal a latent and subvert neurophysiological process not manifest externally when an appropriate task is designed and modelled and given a time - series run to the specific recipient (Kubo \& Nittono, 2008). In sequel, the deception group subject would evince P300 wave form of small amplitude when exposed to irrelevant task as has also been reported by Farwell and Donchin (1991).

The findings of the present study of an increased value of amplitude of P300 wave along the Pz electrode pair supplement and further postscripts the observations and findings of Rosenfeld et al. (2004). The larger amplitude values of P300 wave form on the $\mathrm{Pz}$ electrode pairs suggest that deception entails more conflict and control, coinciding with previous studies using a similar paradigm (Allen, Iacono \& Danielson, 1992). Therefore, deception is a cognitively more demanding process than truth-telling. In order to tell a lie, one has to recognize what is true (the first step) and ensure that the actual response is different from the truthful one (the second step). The second step might result in extra processing time and reduced accuracy (Pfister, 2014). Wu, $\mathrm{Hu} \& \mathrm{Fu}$ in 2009 , for the first time, observed a larger P300 (amplitude and latency) for lying than truth-telling when they asked participants to make deceptive or honest response with an odd ball paradigm. Consistent with most prior work, given 
its spatial-temporal features and sensitivity to deception, the P300 wave form here might represent the conflict detection and response inhibition process, implying that more executive control process is required for deceptive responses (Suchotzki,2015; Wu et al., 2009)

\section{Summary and Conclusion}

It could be concluded from the present study that profiling of P300 wave form, in terms of values of amplitude (in $\mu \mathrm{V}$ ) with appropriate experimental oddball paradigm could represent and act as a supplementary and object toll in the science of Lie Detection and Forensic Medicine.

\section{Recommendations and Limitation}

P300 wave form with adequate and suitable experimental design could be included as an integral part of Detection of Deception and would help agencies involved in investigating the presence or absence of information within the phase space of the working human mind of an alleged suspect.

Large sample size may give more accurate results.

\section{Bibliography}

1. Allen, J. J., Iacono, W. G., \& Danielson, K. D. (1992). The Identification of Concealed Memories Using the Event-Related Potential and Implicit Behavioral Measures: A Methodology for Prediction in the Face of Individual Differences. Psychophysiology, 29(5), 504-522.

2. Donchin, E., \& Coles, M. G. (1988). Is the P300 component a manifestation of context updating. Behavioral and brain sciences, 11(3), 357-427.

3. Farwell, L. A., \& Donchin, E. (1991). The Truth Will Out: Interrogative Polygraphy ("Lie Detection") With Event-Related Brain Potentials. Psychophysiology, 28(5), 531-547.

4. Hillyard, S. A., Hink, R. F., Schwent, V. L., \& Picton, T. W. (1973). Electrical signs of selective attention in the human brain. Science, 182(4108), 177-180.

5. Johnson, R., Jr. (1988). The amplitude of the P300 component of the event-related potential: Review and synthesis. Advances in Psychophysiology, 3, 69-137.

6. Kubo, K., \& Nittono, H. (2008). Detecting the intention to conceal the truth: An event-related potential study. Proceedings, pp, 55, 58 .

7. Pfister, R., Foerster, A. \& Kunde, W. (2014). Pants on fire: The electrophysiological signature of telling a lie. Social Neuroscience, 9, 562-572

8. Rosenfeld, J. P., Soskins, M., Bosh, G., \& Ryan, A. (2004). Simple, effective countermeasures to P300-based tests of detection of concealed information. Psychophysiology, 41(2), 205-219.

9. Suchotzki, K., Crombez, G., Smulders, F. T. Y., Meijer, E. \& Verschuere, B. (2015). The cognitive mechanisms underlying deception: An event-related potential study. International Journal of Psychophysiology 95: 395-405.

10. Vrij, A. (2000). Detecting lies and deceit: The psychology of lying and implications for professional practice. Chichester: John Wiley \& Sons.

11. Wu, H., Hu, X. \& Fu, G. (2009). Does willingness affect the N2-P3 effect of deceptive and honest responses? Neuroscience letters, 467, 63-66. 